\title{
VIOLÊNCIA DOMÉSTICA CONTRA A MULHER EM CONTEXTO RURAL: RECONHECIMENTO DAS ESTRATÉGIAS DE ATENÇÃO
}

\section{Domestic violence against women in rural context: recognition of care strategies
Violencia doméstica contra la mujer en el contexto rural: reconocimiento de las estrategias de atención

\author{
Marta Cocco da Costa (iD \\ Universidade Federal de Santa Maria - UFSM - Palmeira das Missões (RS) - Brasil \\ Ethel Bastos da Silva iD \\ Universidade Federal de Santa Maria - UFSM - Palmeira das Missões (RS) - Brasil \\ Jaqueline Arboit (iD \\ Universidade Federal de Santa Maria - UFSM - Santa Maria (RS) - Brasil \\ Fernanda Honnef (iD \\ Universidade Federal de Santa Maria - UFSM - Santa Maria (RS) - Brasil \\ Karoline Ardenghi Marques (D) \\ Universidade Federal de Santa Maria - UFSM - Palmeira das Missões (RS) - Brasil \\ Janaína Barbieri iD \\ Universidade Federal de Santa Maria - UFSM - Palmeira das Missões (RS) - Brasil \\ Daniela de Mattos da Silva ii \\ Universidade Federal de Santa Maria - UFSM - Palmeira das Missões (RS) - Brasil
}

RESUMO

Objetivo: Descrever a atuação dos profissionais da Estratégia de Saúde da Família (ESF) diante das situações de violência doméstica contra a mulher em contexto rural e os limites enfrentados para o seu desenvolvimento. Métodos: Estudo qualitativo realizado com vinte profissionais de saúde da ESF de dois municípios do estado do Rio Grande do Sul, Brasil. Os dados foram coletados através de entrevistas estruturadas, no período de janeiro a março de 2016. Submeteu-se o material empírico à análise de conteúdo, emergindo as categorias temáticas: atuação dos profissionais da ESF em atenção à mulher em situação de violência doméstica em cenários rurais; limites enfrentados pelos profissionais da ESF para atenção à mulher em situação de violência doméstica em cenários rurais. Resultados: A atenção desenvolvida pelos profissionais da ESF à essas mulheres relacionam-se com a escuta atenta e sensível e trabalho em equipe e intersetorial. Os limites para o desenvolvimento da atuação dos profissionais são a falta de habilidade, a não participação em grupos, negação da vivência da situação de violência e a ausência de um protocolo para nortear as ações dos profissionais diante da identificação da violência. Conclusão: Apesar de as estratégias de atenção às mulheres rurais em situação de violência doméstica pautarem-se nos pressupostos da humanização e promoção da saúde, existem limites para a atenção integral dessas mulheres. Sugere-se a criação de um modelo que priorize a capacitação dos profissionais para atuar com a mulher em situação de violência e um protocolo para nortear as ações diante de tal situação.

Descritores: Violência Doméstica; Violência contra a Mulher; Estratégia Saúde da Família; Pessoal de Saúde; Zona Rural; Saúde da População Rural.

\section{ABSTRACT}

Objective: Describe the role of Family Health Strategy (FHS) professionals in the face of domestic violence against women in a rural context and the limits faced for their development. Methods: Qualitative study conducted with twenty FHS health professionals from two municipalities of the state of Rio Grande do Sul, Brazil. Data were collected through structured interviews from January to March 2016. The empirical material was submitted to content analysis, emerging the thematic categories: performance of

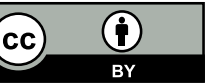


FHS professionals in attention to women in situations of domestic violence in rural settings; limits faced by FHS professionals to care for women in situations of domestic violence in rural settings. Results: The attention developed by the FHS professionals to these women is related to attentive and sensitive listening and teamwork and intersectoral. The limits for the development of the professionals 'performance are the lack of skill, non-participation in groups, denial of the experience of the violence situation and the absence of a protocol to guide the professionals' actions regarding the identification of violence. Conclusion: Although the strategies of attention to rural women in situations of domestic violence are based on the assumptions of humanization and health promotion, there are limits to the integral care of these women. It is suggested to create a model that prioritizes the training of professionals to work with women in situations of violence and a protocol to guide the actions in the face of such a situation.

Descriptors: Domestic Violence; Violence Against Women; Family Health Strategy; Health Personnel; Rural Areas; Rural Health.

\section{RESUMEN}

Objetivo: Describir la actuación de los profesionales de la Estrategia de Salud de la Familia (ESF) ante situaciones de violencia doméstica contra la mujer en el contexto rural y los límites afrontados para su desarrollo. Métodos: Estudio cualitativo realizado con veinte profesionales sanitarios de la ESF de dos municipios del estado de Rio Grande do Sul, Brasil. Se recogieron los datos a través de entrevistas estructuradas en el período entre enero y marzo de 2016. Se ha realizado un análisis de contenido del material empírico del cual ha emergido las siguientes categorías temáticas: actuación de los profesionales de la ESF para la atención de la mujer en situación de violencia doméstica en escenarios rurales; límites afrontados por los profesionales de la ESF para la atención de la mujer en situación de violencia doméstica en escenarios rurales. Resultados: La atención desarrollada por los profesionales de la ESF a esas mujeres se relacionan con la escucha atenta y sensible y el trabajo en equipo e intersectorial. Los límites para el desarrollo de la actuación de los profesionales son la falta de habilidad, la no participación en grupos, la negación de la vivencia de situación de violencia y la ausencia de un protocolo para orientar las acciones de los profesionales ante la identificación de violencia. Conclusión: Pese a que las estrategias para la atención de las mujeres rurales en situación de violencia doméstica se basan en los presupuestos de la humanización y la promoción de la salud, hay límites para la atención integral de esas mujeres. Sugiérase la creación de un modelo que valore la capacitación de los profesionales para actuar con la mujer en situación de violencia y un protocolo para direccionar las acciones ante tal situación.

Descriptores: Violencia Doméstica; Violencia contra la Mujer; Estrategia de Salud Familiar; Personal de Salud; Medio Rural; Salud Rural.

\section{INTRODUÇÃO}

A violência tem se tornado um problema de saúde pública, pois atinge a sociedade de modo geral e repercute em agravos à saúde de quem a vivencia ${ }^{(1,2)}$. Deve ser considerada um fenômeno complexo, de proporção mundial, com tendência de crescimento nas populações devido à sua magnitude ${ }^{(3)}$.

Em relação às mulheres, de acordo com a Organização Mundial da Saúde (OMS) ${ }^{(2)}$, aproximadamente $30 \%$ delas já sofreram algum tipo de violência por parte de seu parceiro íntimo. No Brasil, $29 \%$ das mulheres, ao longo da sua vida, vivenciaram alguma situação de violência( ${ }^{(4)}$.

Na questão da violência doméstica, destaca-se que é um fenômeno de determinações múltiplas, sendo definida como qualquer ação ou omissão perpetrada no ambiente doméstico por indivíduos que convivam neste, com ou sem função parental, mesmo que eventualmente ${ }^{(5)}$. Esse termo denota a violência em suas distintas tipologias e pode ser direcionada a diferentes populações, como mulheres, crianças e adolescentes ${ }^{(6)}$.

Quando se tratam de situações de violência no contexto rural, estas se potencializam. Isto se associa à vida e ao trabalho das pessoas que vivem nesse contexto, bem como ao fato de que esse ambiente está localizado distante dos grandes centros e, consequentemente, dos recursos sociais, políticos e comunitários que poderiam promover maior proteção ${ }^{(7-10)}$. Nesse cenário, a violência doméstica é permeada por condições sociodemográficas, econômicas e culturais, e sustentada pela hierarquia de poder e dominação empregados nas relações intrafamiliares, intersexuais e intergeracionais ${ }^{(11,12)}$.

Desse modo, esses aspectos devem ser considerados para o êxito da atenção às mulheres em situação de violência doméstica residentes em cenários rurais, o que está em concordância com os princípios de promoção da saúde. Esta, conforme a OMS, consiste em proporcionar à população os meios necessários para qualificar a sua saúde e exercer controle sobre a mesma, a partir de programas e políticas balizados nos princípios de concepção humanista, participação social e equidade ${ }^{(13)}$.

$\mathrm{Na}$ tentativa de superar a fragmentação da atenção às mulheres em situação de violência, para que seja orientada pela perspectiva da integralidade, publicaram-se políticas para suprir as lacunas na atenção às mulheres, como a 
Política Nacional de Atenção Integral à Saúde da Mulher (PNAISM), que incorpora a integralidade na perspectiva de gênero para alcançar a promoção e a humanização da saúde como princípios norteadores ${ }^{(14)}$. Em 2006, publicouse a Política Nacional de Promoção da Saúde, por meio da qual se incorporaram inúmeras práticas em todas as esferas do governo e entendeu-se que, para a promoção da saúde, é imprescindível intervir em problemas como a violência contra a mulher, por ser a um agravante a sua saúde, entre outros ${ }^{(15)}$.

Nesse cenário, a Estratégia Saúde da Família (ESF) é o serviço de saúde que tende a estar mais próximo dos indivíduos em situações de violência. A ESF propõe ações de cuidado que permitem a identificação de problemas sociais, entre eles, a violência doméstica, bem como o desenvolvimento de respostas a esses problemas na perspectiva da integralidade ${ }^{(16)}$. Além disso, destaca-se pela possibilidade de continuidade e de acompanhamento das ações iniciadas após a identificação das situações de violência doméstica ${ }^{(17)}$.

Ressalta-se a importância de estratégias de atenção que visem ao cuidado longitudinal e integral das mulheres que vivenciam situações de violência doméstica. No entanto, para garantir uma atenção qualificada, faz-se necessário conhecimento e preparo dos profissionais que as assistam, com ações efetivas e resolutivas, em especial aqueles atuantes na $\operatorname{ESF}^{(18)}$.

Com base no exposto, a relevância deste estudo consiste em descrever a atuação dos profissionais de saúde diante das situações de violência doméstica contra a mulher em contexto rural e os limites por eles considerados, a fim de servir de subsídios a outros estudos. Proposta que converge com os temas de pesquisa da Agenda Nacional de Prioridades de Pesquisa em Saúde ${ }^{(19)}$ e com a agenda de Desenvolvimento Sustentável, que aponta como um de seus objetivos a redução significativa de todas as formas de violência em todos os lugares ${ }^{(20)}$.

Assim, o presente estudo tem a seguinte questão norteadora: qual a atuação dos profissionais da Estratégia de Saúde da Família frente às situações de violência doméstica em contexto rural e aos limites para desenvolvê-las? Para respondê-la, tem-se como objetivo descrever a atuação dos profissionais da Estratégia de Saúde da Família diante das situações de violência doméstica contra a mulher em contexto rural, e os limites enfrentados para o seu desenvolvimento.

\section{MÉTODOS}

Estudo de abordagem qualitativa, o que apresenta coerência com o objeto de estudo, visto que é empregada ao se abordar o estudo da história, das relações, das representações, das crenças, das percepções e das opiniões, as quais resultam da interpretação humana acerca de suas vivências e sentimentos ${ }^{(21)}$.

O estudo teve como cenário dois municípios da região Noroeste do estado do Rio Grande do Sul, Brasil. Para elencar esses municípios, buscaram-se região com população rural maior que a urbana e com Estratégia da Saúde da Família nos cenários rurais. Assim, elencaram-se quatro municípios como convidados para participar da pesquisa, obtendo-se resposta positiva de dois desses municípios.

Os participantes do estudo constituem todos os profissionais (enfermeiros, técnicos de enfermagem, agentes comunitários de saúde, cirurgiões-dentistas e auxiliares de saúde bucal) que atuavam junto às ESF em cenários rurais. Uma vez que esses profissionais atendem, em algum momento, mulheres em situação de violência, poderiam, então, detalhar as especificidades da atuação e os limites na atenção às mulheres em situações de violência doméstica nos cenários rurais.

A geração dos dados iniciou com a aproximação da pesquisadora ao cenário de estudo. Essa estratégia permitiu apresentar o estudo, negociar como poderia ser realizada a entrevista, escolher o local e convidar os profissionais a participar do estudo. Depois, conforme a disponibilidade dos profissionais, agendou-se o dia e o horário para realização das entrevistas.

Para a coleta de dados, utilizou-se as entrevistas estruturadas que pressupõem perguntas previamente formuladas, caracterizadas por serem abertas ${ }^{(21)}$. As questões pautaram-se na atenção dos profissionais prestada às mulheres em situação de violência nos cenários rurais, com enfoque na atuação e nos limites dessa atenção. Qual sua atuação diante de uma situação de violência doméstica à mulher no seu local de trabalho rural? Quais os limites encontrados na atenção à mulher nas situações de violência doméstica no seu local de trabalho rural?

As entrevistas ocorreram individualmente, conforme prévio agendamento, no período de janeiro de 2016 a março de 2016, sendo realizadas em sala reservada no próprio serviço em que trabalhavam. Para garantir a fidedignidade, as entrevistas foram gravadas em áudio, com o consentimento dos participantes, com duração média de 30 minutos.

O recrutamento de participantes encerrou-se mediante o critério de saturação temática(22), alcançado no $20^{\circ}$ profissional. Dentre esses profissionais, destacam-se: três enfermeiros, três técnicos de Enfermagem, onze agentes 
comunitários de saúde, dois cirurgiões-dentistas e um auxiliar de saúde bucal. Como critério de inclusão, elencou-se: atuar há pelo menos seis meses nesses serviços. O critério de exclusão compreendeu estar em período de férias ou de licença de qualquer natureza no período de coleta de dados. Cinco profissionais de um mesmo município optaram por não participar.

As falas dos entrevistados foram submetidas à análise de conteúdo após a transcrição. O período de análise dividiu-se em três etapas: pré-análise, exploração do material, e tratamento dos resultados obtidos e interpretação(21). Quanto à pré-análise, esta se iniciou com a organização do material obtido mediante as entrevistas para posterior análise. Para tanto, primeiramente foi realizada a transcrição, na íntegra, dos áudios das entrevistas em um editor de textos, compondo o corpus do estudo. Em seguida, realizou-se a escuta das gravações e a leitura flutuante, que possibilitou gerar impressões iniciais acerca do material ${ }^{(21)}$. Depois, realizou-se uma sequência de leituras exaustivas, que permitiram destacar, com cores distintas, os fragmentos nos quais os depoimentos dos participantes eram correlatos $^{(21)}$. A exploração do material se deu mediante o recorte das informações comuns encontradas no material transcrito. Nesse sentido, elencaram-se os núcleos de sentido, os quais se referem a palavras, frases e expressões que dão sentido ao conteúdo das falas e subsidiam a constituição das categorias(21).

Aúltima fase constitui-se no tratamento dos resultados obtidos e interpretação. Nessa fase, fez-se inferências e interpretações acerca dos resultados, sempre com retorno ao objetivo do estudo ${ }^{(21)}$. Em seguida, foram agrupados, permitindo a constituição de duas categorias temáticas: atuação dos profissionais da ESF em atenção à mulher em situação de violência doméstica em cenários rurais; limites enfrentados pelos profissionais da ESF para atenção à mulher em situação de violência doméstica em cenários rurais.

Realizou-se este estudo de forma a respeitar as normas da Resolução n. ${ }^{\circ} 466 / 12$ do Conselho Nacional de Saúde ${ }^{(23)}$, apreciado e aprovado pelo Comitê de Ética em Pesquisa da Universidade Federal do Rio Grande do Sul, sob Parecer n. ${ }^{\circ}$ 514.865. Destaca-se que, antes do procedimento de coleta de dados, esclareceu-se aos participantes, individualmente, por meio do Termo de Consentimento Livre e Esclarecido, acerca dos objetivos da pesquisa. Haja vista o anonimato dos participantes, utilizou-se o código $\mathrm{P}$ de profissional, seguido do número que representa a sequência da realização da entrevista.

\section{RESULTADOS E DISCUSSÃO}

\section{Atuação dos profissionais da ESF em atenção à mulher em situação de violência doméstica em cenários rurais}

A categoria temática descreve a atuação dos profissionais da ESF em atenção à mulher em situação de violência doméstica em cenários rurais, na qual os profissionais mencionaram elementos essenciais para compreender e dar visibilidade para as situações de violência, assim como a observação e a escuta atenta e sensível realizadas durante os atendimentos e na visita domiciliar:

\section{"[...] o nosso trabalho é mais de escuta." (P1) \\ "[...] minha atuação é conversar." (P4) \\ "[...] na maioria dos casos, eu procuro escutar, observar, para tentar entender." (P5)}

Ao escutar atentamente e sensivelmente as mulheres que vivenciam situações de violência doméstica no cenário rural, os profissionais demonstraram interesse por compreender as situações e, com isso, as necessidades de quem as vivencia. Nessa direção, entende-se que é necessário que os profissionais se mantenham sempre atenciosos e disponíveis para ouvir as mulheres em situação de violência, desenvolvendo uma escuta não julgadora ${ }^{(24)}$. Tal conduta auxiliará no estabelecimento de vínculo e confiança entre as mulheres e os profissionais, facilitando a construção compartilhada de estratégias de enfrentamento/superação da situação.

Normalmente, as mulheres em situação de violência doméstica no cenário rural relatam sua situação aos profissionais quando já possuem vínculo e confiança neles, como mencionado pelos entrevistados:

"[...] depois de um tempo que tu frequentas a casa, elas conseguem se abrir [...] primeiro tem que conseguir a confiança da família, para depois ela te colocar os problemas." (P1)

"Muitas vezes, elas choram ou se queixam [...] então, conversando, com o passar do tempo, elas vão contando o que está acontecendo e a gente vai também desenvolvendo empatia [...]" (P15)

"[...] em uma visita tu percebes, através da observação, que não estão bem e, depois, numa outra visita, eles te contam, 'oh aquele dia eu estava assim, porque aconteceu isso e isso'." (P19) 
Destaca-se, pelas falas, que o vínculo e a confiança são decorrentes da maneira como os usuários são tratados pelos profissionais. Também tem a ver com o tempo de convívio com o usuário, pois não é no primeiro contato com o profissional, seja na unidade de saúde, seja no domicílio, que a situação de violência será relatada. Assim, quando os profissionais demonstram empatia, as mulheres sentem-se mais à vontade para relatar as situações. A importância da confiança e vínculo entre a usuária e o profissional, a fim de romper o estigma e viabilizar o relato da violência, também é exposta em estudo que investigou as ações realizadas por enfermeiros da atenção primária em saúde para mulheres em situação de violência doméstica ${ }^{(25)}$.

Outra atuação mencionada pelas entrevistadas centrou-se sobre os programas de rádio e algum programa de televisão que falou sobre o assunto, não banalizando a violência. Assim, viu-se o tema violência doméstica, discutido nos programas de rádio e televisão com o intuito de levar as mulheres a compreenderem o que é, mencionado pelos entrevistados:

"[...] sempre tem algum programa no rádio, na televisão que eles explicam e orientam as pessoas, e a gente tenta, também, orientar para as pessoas perceberem e falarem sobre seu problema." (P19)

"[...] tentar abordar mais o assunto, orientando, explicando [...] porque acho que, às vezes, as pessoas nem sabem que aquilo não é normal [...] de repente, dentro de casa, tu vives aquela situação a vida inteira e acha que aquilo é normal." (P9)

"[...] a gente quase não vê, a gente só imagina que aquilo é uma violência, [...] é através das visitas que a gente nota." (P3)

Contudo, pelas falas, reflete-se que a pobreza esteja articulada na construção das violências para este recorte populacional rural. Admite-se, então, que mulheres rurais pobres possuem menores probabilidades de exercício da cidadania, de luta pelos seus direitos, verificada, por exemplo, na ausência ou limitação da autonomia financeira para sustentar-se ou mesmo para percorrer as enormes distâncias entre as suas residências e os serviços estatais de atenção, acolhimento e controle social, incomuns no interior dos estados, sendo predominantemente urbanos ${ }^{(26)}$.

Quanto às visitas domiciliares, outra forma de atuação, é que os profissionais podem observar, por ventura, lesões nessas mulheres e o próprio ambiente da casa, que pode denunciar algo, bem como obter informações acerca das relações familiares no ambiente doméstico ${ }^{(27)}$. Isto pode caracterizar a vivência de alguma forma de violência doméstica. Nas visitas, destaca-se o trabalho do agente comunitário de saúde, cuja principal atribuição é a realização dessa atividade. Assim, esse profissional age como interlocutor das situações de violência doméstica à equipe de saúde, pela proximidade com os usuários, porque atua diretamente com as famílias e, com isso, possivelmente possui uma relação de vínculo mais consolidada(28).

Os resultados da investigação em tela corroboram o estudo que evidenciou que a equipe da ESF, quando suspeita/confirma situações de violência, contata frequentemente o ACS em decorrência do conhecimento que esse profissional detém acerca do cotidiano das famílias em suas áreas de abrangência. Esse profissional pode advertir a equipe acerca dos riscos ou fracassos em relação a alguma ação planejada, auxiliar na decisão pela melhor abordagem (consulta ou visita domiciliar) para acessar a família, acompanhar os casos e os resultados das intervenções desenvolvidas, bem como reunir informações de vizinhos e familiares ${ }^{(6)}$.

Os profissionais relataram atuação na perspectiva coletiva do trabalho em equipe, como a discussão e o planejamento de casos:

"[...] A gente coloca isso para o grupo: agentes comunitários de saúde, técnicos, enfermeiros e médicos e, numa ação conjunta, vamos tentar achar uma solução." (P1)

"[...] Quando eles (agentes comunitários de saúde) trazem para mim a realidade de um paciente, eu pergunto qual é a forma que acham que a gente deve trabalhar. Então, eu não planejo uma coisa sozinha; na verdade, fazemos o planejamento em equipe." (P6)

A atuação compartilhada da equipe, mediante discussão de casos, por exemplo, é vista como uma forma de apoio entre os profissionais na atenção aos usuários em situação de violência doméstica no contexto rural. Essa forma de atuação, relatada em outros estudos, tende a integrar os pontos de vista de diferentes profissionais, ao mesmo tempo em que instiga a corresponsabilização entre os membros da equipe para que juntos construam estratégias para enfrentamento da violência em seus territórios de atuação( ${ }^{(6,29,30)}$. Para tanto, deve-se considerar e respeitar as competências de cada profissional para o êxito no cuidado integral dos usuários nessas situações.

Contudo, devido à complexidade da problemática, nem sempre a ESF rural consegue amparar suficientemente os usuários em situações de violência doméstica, por isso é importante o suporte de profissionais e serviços da rede intersetorial: 
"[...] A gente coloca isso para o grande grupo [...] temos psicólogas, tem também apoio da assistente social que é o CRAS [Centro de Referência de Assistência Social] e CREAS [Centro de Referência Especializado de Assistência Social]." (P1)

"[...] dependendo do caso, a gente repassa para a psicóloga e para assistente social do NASF [Núcleo de Apoio à Saúde da Família] e NAAB [Núcleo de Apoio à Atenção Básica]." (P11)

O profissional que realiza o primeiro contato com o usuário, seja no domicílio ou na unidade de ESF rural, tem a possibilidade de evidenciar quais os profissionais da equipe e/ou de outros serviços poderão contribuir no enfrentamento da situação de violência. No que se refere à necessidade de contatar profissionais da rede intersetorial, os participantes relataram que acionam o Centro de Referência e os Centros de Apoio. Essa iniciativa reitera que problemas complexos, como a violência doméstica, requerem para seu enfrentamento a articulação de diferentes setores, com interdependência entre si.

No que concerne, especificamente, acerca da participação dos profissionais do NASF (psicólogos e assistentes sociais) em discussão de casos de violência, estudo apontou que essa participação implica em maior celeridade e respostas positivas, tanto pela possibilidade de desenvolver atividades do seu núcleo como avaliação psicológica quanto pela interlocução entre os diversos saberes e fazeres dos profissionais envolvidos no enfrentamento das situações de violência(6).

\section{Limites enfrentados pelos profissionais da ESF para atenção à mulher em situação de violência doméstica em cenários rurais}

Nesta categoria temática são descritos os limites enfrentados pelos profissionais da ESF para atenção à mulher em situação de violência doméstica em cenários rurais. Um deles se refere à falta de habilidade dos profissionais para atuar no reconhecimento e enfrentamento dessas situações:

\section{"[...] a gente poderia fazer mais, desde que tivéssemos mais conhecimento." (P5) \\ "[...] fica difícil tomar uma conduta... pois não sabemos, muitas vezes, como agir..." (P4)}

Em estudo desenvolvido com enfermeiros sobre essa temática, foi citada a falta de formação profissional para o reconhecimento e resolução de situações de violência frente à complexidade desse problema ${ }^{(25)}$. O despreparo dos profissionais, tanto durante a graduação quanto nos serviços, leva-os a desenvolverem condutas distantes do que é recomendado para uma atenção qualificada nos casos de violência doméstica ${ }^{(31)}$. Diante disso, faz-se premente o desenvolvimento de atividades de educação permanente com esses profissionais, a fim de sensibilizálos e capacitá-los para atuar com usuários que vivem em situação de violência, especialmente no cenário rural, com base na distância geográfica e na ausência/escassez de recursos (profissionais e serviços) nesse cenário.

Além disso, os profissionais também referiram como limites a falta de participação das mulheres em situação de violência nos grupos para as quais são convidadas. Um dos motivos pelos quais as pessoas não participam dos grupos, de acordo com os profissionais, é a falta de meio de transporte para chegar até a ESF e/ou ausência de recursos financeiros para pagar transporte coletivo:

"Tem muitos programas, só que muitos não participam [...]" (P3)

"A gente tem programas, tem reuniões, tem encontros, não participam de nada." (P6)

"Às vezes, tem uma reunião que eles poderiam participar, só que como é longe, para virem de ônibus não têm condições de pagar a passagem [...] porque aquele dinheiro faz falta para comprar a comida." (P19)

O fato de os usuários não participarem das atividades grupais oferecidas pela ESF rural também demonstrou ser um fator limitante para a discussão dessa situação, pois, nesses espaços, embora não se direcionem especificamente às mulheres em situação de violência, pode-se abordar a problemática da violência doméstica como um dos primeiros passos para o seu enfrentamento.

Diante desse limite, os profissionais expuseram que muitos usuários não conseguem participar dos grupos por não terem condições financeiras para pagar transporte coletivo ou pela ausência desse tipo de transporte ou mesmo de meio de transporte próprio para deslocarem-se até os locais onde os grupos ocorrem. Posto isso, os profissionais devem buscar formas para se aproximar geograficamente dos usuários. Uma delas seria a utilização dos recursos disponíveis nos cenários rurais, como salões comunitários, escolas ou outros espaços da comunidade rural. Assim, diante dessa dificuldade, sugere-se desenvolver atividades educativas de sala de espera com as mulheres quando em estejam na unidade aguardando consultas. 
Outro limite citado pelos profissionais é a negação das situações de violência por quem a vivencia:

"Na verdade, a maioria delas nega. A gente sabe pela feição, pelo físico [...] uma pessoa que sempre está cabisbaixa. Então, a gente acaba diagnosticando por isso, mas quando a gente fala no assunto: 'Não! Não acontece nada, eu que sou assim, é o meu jeito' [...] Tem muita negação." (P6)

"A dificuldade é fazer elas [pessoas em situação de violência doméstica] contar, admitir que sofrem [...]." (P14)

A negação da vivência das situações de violência doméstica também constitui um limite para a superação dessas situações. Apesar de os profissionais identificarem pela fisionomia e pelas expressões das mulheres, quando as questionam sobre a vivência da violência, elas negam e, com isso, limitam a possibilidade de ações para enfrentamento desse problema. Isto pode se dar tanto pelo medo de expor a situação como pela naturalização da problemática no meio rural, cenário no qual, historicamente, as mulheres ocupam posições subalternas na hierarquia familiar ${ }^{(32)}$.

Os profissionais também citaram a falta de um protocolo na ESF a ser seguido diante do reconhecimento das situações de violência, o que limita o desenvolvimento de estratégias de atenção:

"Às vezes, sim, são discutidas [se referindo as situações de violência doméstica], mas não tem um plano para a gente agir. Por onde eu vou chegar, o que eu vou falar." (P5)

Apesar de os participantes relatarem que, por vezes, identificam as situações de violência, citaram a falta de um protocolo, situação limitante para implementação de ações com as mulheres nesta situação; corroborando com outro estudo, no qual a fragilidade de recursos tecnológicos, diante das situações de violência, leva os profissionais a realizarem suas práticas assistenciais em protocolos, os quais explicitam a ordem de ações a serem desenvolvidas ${ }^{(33)}$. Acredita-se que é importante a institucionalização de documentos, a exemplo de protocolos, a fim de orientar e dar suporte na assistência às pessoas em situações de violência doméstica.

O presente estudo permite visualizar as estratégias de atenção e os limites encontrados, favorecendo pensar em ações que permitam fortalecer programas de saúde, como o de Promoção da saúde, contribuindo para a construção de uma assistência mais humanizada, com uma abordagem diferenciada da visão tradicional do modelo biomédico. Nessa perspectiva, a transversalidade da Política Nacional de Promoção da Saúde (PNPS) na atenção às mulheres em situação de violência em cenário rural contempla agregar o tema em redes que propiciem práticas de cuidado humanizadas, que promovam o diálogo e construam práticas amparadas na integralidade do cuidado e da saúde ${ }^{(15,34)}$.

Como implicações para a prática clínica, este estudo fornece elementos essenciais para orientar a capacitação dos profissionais, seja na graduação, seja nos serviços de atenção à pessoas em situação de violência doméstica residentes em áreas rurais. Recomenda-se o desenvolvimento de estudos de natureza interventiva, a fim de potencializar as ações já desenvolvidas e propor estratégias para a superação dos limites existentes. Novas pesquisas podem ser desenvolvidas com o objetivo de fornecer subsídios para superação dos limites apontados pelos profissionais durante a atenção às mulheres em situação de violência doméstica em cenários rurais.

Este estudo apresenta limitações inerentes a estudos qualitativos, em especial no que se refere à investigação de municípios de uma única região do estado do Rio Grande do Sul, cujas condições dificultam a generalização dos resultados.

\section{CONSIDERAÇÕES FINAIS}

Os profissionais investigados desenvolvem com as mulheres rurais em situação de violência estratégias de atenção, como a observação, a escuta atenta e sensível, o vínculo, a confiança, as orientações, a visita domiciliar, o trabalho com a equipe da ESF rural e nos demais serviços disponíveis na rede de atenção intersetorial.

Dentre os limites para desenvolver tais estratégias, encontraram a falta de habilidade, a não participação das mulheres nos grupos, a negação da vivência da situação de violência e a ausência de um protocolo para nortear as ações dos profissionais diante da identificação da violência.

Sugere-se a criação de um modelo que priorize a capacitação dos profissionais de saúde para atuar com a mulher em situação de violência e um protocolo para nortear as ações diante de tal situação.

\section{CONFLITOS DE INTERESSE}

Os autores declaram que não houve conflitos de interesses. 


\section{CONTRIBUIÇÕES}

Marta Cocco da Costa e Ethel Bastos da Silva contribuíram com a elaboração e delineamento do estudo; a aquisição, análise e interpretação de dados; e a redação e/ou revisão do manuscrito. Jaqueline Arboit, Fernanda Honnef, Karoline Ardenghi Marques, Janaína Barbieri e Daniela de Mattos da Silva contribuíram com a elaboração e delineamento do estudo e a redação e/ou revisão do manuscrito.

\section{REFERÊNCIAS}

1. Donoso MTV, Bastos MAR. O cotidiano dos profissionais que trabalham diretamente com vítimas de violência social. Rev Enferm Cent-Oeste Min [Internet]. 2014 [acesso em 2018 Mar 22];4(1):951-60. Disponível em:http://www.seer.ufsj.edu.br/index.php/recom/article/view/423/570

2. World Health Organization. Global and Regional estimates of violence against women: prevalence and health effects of intimate partner violence and non-partner sexual violence [Internet]. Geneva: WHO; 2013 [acesso em 2018 Mar 10]. Disponível em: http://apps.who.int/iris/bitstream/handle/10665/85239/9789241564625_eng. pdf;jsessionid=2C27540FD7EC0064EB0B57A9305A694E? sequence $=1$

3. García-Moreno C, Zimmerman C, Morris-Gehring A, Heise L, Amin A, Abrahams N, et al. Addressing violence against women: a call to action. Lancet [Internet]. 2015 [acesso em 2018 Mar 01];385(9978):1685-95. Disponível em: https://linkinghub.elsevier.com/retrieve/pii/S0140-6736(14)61830-4

4. Brasil. Senado Federal. DataSenado. Violência doméstica e familiar contra a mulher [Internet]. Brasília: Senado Federal; 2017 [acesso em 2018 Nov 22]. Disponível em: https://www12.senado.leg.br/institucional/ datasenado/arquivos/aumenta-numero-de-mulheres-que-declaram-ter-sofrido-violencia

5. Ministério da Saúde (BR). Secretaria de Políticas de Saúde. Violência intrafamiliar: orientações para prática em serviço [Internet]. Brasília: Ministério da Saúde; 2001 [acesso em 2018 Dez 05]. Disponível em: http:// bvsms.saude.gov.br/bvs/publicacoes/cd05_19.pdf

6. Moreira TNF, Martins CL, Feuerwerker LCM, Schraiber LC. The foundation of care: Family Health Program teams dealing with domestic violence situations. Saúde Soc [Internet]. 2014 [acesso em 2018 Jun 01];23(3):814-27. Disponível em: http://www.scielo.br/pdf/sausoc/v23n3/en_0104-1290-sausoc-23-3-0814.pdf

7. Costa MC, Silva EB, Soares JSF, Borth LC, Honnef F. Rural women and violence situation: access and accessibility limits to the healthcare network. Rev Gaúch Enferm [Internet]. 2017 [acesso em 2018 Jun 03];38(2):e59553. Disponível em: http://www.scielo.br/pdf/rgenf/v38n2/en_0102-6933rgenf-1983-144720170259553.pdf

8. Benson SR. Assisting rural domestic violence victims: the local librarian's role. Law Libr J [Internet]. 2016 [acesso em 2018 Jun 02];108(2):237-50. Disponível em: https://www.ideals.illinois.edu/bitstream/ handle/2142/90427/LLJ_108n2_04_benson.pdf?sequence=2

9. Peek-Asa C, Wallis A, Harland K, Beyer K, Dickey P, Saftlas A. Rural disparity in domestic violence prevalence and access to resources. J Womens Health [Internet]. 2011 [acesso em 2018 Jun 02];20(11):1743-9. Disponível em: https://www.ncbi.nlm.nih.gov/pmc/articles/PMC3216064/pdf/jwh.2011.2891. pdf

10. Dudgeon A, Evanson TA. Intimate partner violence in rural U.S. areas: what every nurse should know. Am J Nurs [Internet]. 2014 [acesso em 2018 Jun 02];114(5):26-35. Disponível em: https://www.nursingcenter.com/ pdfjournal?AID=2446051\&an=00000446-201405000-00023\&Journal_ID=54030\&Issue_ID=2445975

11. Bandeira LM. Violência de gênero: a construção de um campo teórico e de investigação. Rev Soc Estado [Internet]. 2014 [acesso em 2018 Jun 04];29(2):449-69. Disponível em: http://www.scielo.br/pdf/se/v29n2/08. pdf

12. Sá SD, Werlang BSG. Personalidade de mulheres vítimas de violência doméstica: uma revisão sistemática da literatura. Contextos Clín [Internet]. 2013 [acesso em 2018 Jun 04];6(2):106-16. Disponível em: http:// pepsic.bvsalud.org/pdf/cclin/v6n2/v6n2a05.pdf

13. World Health Organization. The Ottawa charter for health promotion. Washington: WHO; 1996. 
14. Ministério da Saúde (BR). Secretaria de Atenção à Saúde. Política nacional de atenção integral à saúde da mulher: princípios e diretrizes, Departamento de Ações Programáticas Estratégicas [Internet]. Brasília: Ministério da Saúde; 2004 [acesso em 2018 Jun 10]. Disponível em: http://bvsms.saude.gov.br/bvs/ publicacoes/politica_nac_atencao_mulher.pdf

15. Ministério da Saúde (BR). Secretaria de Vigilância em Saúde, Secretaria de Atenção à Saúde. Política Nacional de Promoção da Saúde: revisão da Portaria MS/GM nº 687, de 30 de março de 2006 [Internet]. Brasília: Ministério da Saúde; 2015 [acesso em 2018 Jul 14]. Disponível em: http://bvsms.saude.gov.br/bvs/ publicacoes/pnps_revisao_portaria_687.pdf

16. Guedes RB, Fonseca RMGS, Egry EY. The evaluative limits and possibilities in the Family Health Strategy for gender-based violence. Rev Esc Enferm USP [Internet]. 2013 [acesso em 2018 Jun 04];47(2):303-9. Disponível em: http://www.scielo.br/pdf/reeusp/v47n2/en_05.pdf

17. Porto RTS, Bispo JP Júnior, Lima EC. Violência doméstica e sexual no âmbito da Estratégia de Saúde da Família: atuação profissional e barreiras para o enfrentamento. Physis [Internet]. 2014 [acesso em 2018 Jun 07];24(3):787-807. Disponível em: http://www.scielo.br/pdf/physis/v24n3/0103-7331-physis-24-03-00787.pdf

18. Acosta DF, Gomes VLO, Oliveira DC, Gomes GC, Fonseca AD. Ethical and legal aspects in nursing care for victims of domestic violence. Texto \& Contexto Enferm [Internet]. 2017 [acesso em 2018 Maio 12];26(3):e6770015. Disponível em: http://www.scielo.br/pdf/tce/v26n3/en_0104-0707-tce-26-03-e6770015. pdf

19. Ministério da Saúde (BR). Secretaria de Ciência, Tecnologia e Insumos Estratégicos. Agenda Nacional de prioridades de Pesquisa em Saúde [Internet]. Brasília: Ministério da Saúde; 2015 [acesso em 2018 Nov 10]. Disponível em: http://brasil.evipnet.org/wp-content/uploads/2017/07/ANPPS.pdf

20. United Nations General Assembly. Transforming our world: the 2030 Agenda for Sustainable Development [Internet]. New York: UNGA; 2015 [acesso em 2018 Mar 12]. Disponível em: http://www.un.org/ga/search/ view_doc.asp?symbol=A/RES/70/1\&Lang=E

21. Minayo MCS. O desafio do conhecimento: pesquisa qualitativa em saúde. 14ª ed. São Paulo: Hucitec; 2014.

22. Fontanella BJB, Ricas J, Turato ER. Amostragem por saturação em pesquisas qualitativas em saúde: contribuições teóricas. Cad Saúde Pública [Internet]. 2008 [acesso em 2018 Maio 14];24(1):17-27. Disponível em: http://www.scielo.br/pdf/csp/v24n1/02.pdf

23. Ministério da Saúde (BR). Conselho Nacional de saúde. Resolução $n^{\circ} 466 / 2012$. Normas para pesquisa envolvendo seres humanos [Internet]. Brasília: Ministério da Saúde; 2012 [acesso em 2018 Dez 01]. Disponível em: http://conselho.saude.gov.br/resolucoes/2012/reso466.pdf

24. Zuchi CZ, Silva EB, Costa MC, Arboit J, Fontana DGR, Honnef F, et al. Violence against women: conceptions of family health strategy professionals about listening. REME Rev Min Enferm [Internet]. 2018 [acesso em 2018 Jun 09];22:e-1085. Disponível em: http://www.reme.org.br/artigo/detalhes/1223

25. Visentin F, Vieira LB, Trevisan I, Lorenzini E, Silva EF. Women's primary care nursing in situations of gender violence. Invest Educ Enferm [Internet]. 2015 [acesso em 2018 Jun 01];33(3):556-64. Disponível em: http:// www.scielo.org.co/pdf/iee/v33n3/v33n3a20.pdf

26. Bueno ALM, Lopes MJM. Mulheres rurais e violências: leituras de uma realidade que flerta com a ficção. Ambiente Soc. 2018;21:1-22.

27. Heisler ED, Silva EB, Costa MC, Jahn AC, Arboit J. Potential and limits of home visits to identify and address women in situation of violence. Ciênc Cuid Saúde [Internet]. 2017 [acesso em 2018 Jun 09];16(3):1-8. Disponível em: http://periodicos.uem.br/ojs/index.php/CiencCuidSaude/article/view/35348/21071

28. Costa KG, Reichert LP, França JRFS, Collet N, Reichert APS. Concepções e práticas dos profissionais de saúde acerca da violência intrafamiliar contra crianças e adolescentes. Trab Educ Saúde [Internet]. 2015 [acesso em 2018 Jun 09];13(2):79-95. Disponível em: http://www.scielo.br/pdf/tes/v13s2/1981-7746-tes13-s2-0079.pdf

29. Machado JC, Rodrigues VP, Vilela ABA, Simões AV, Morais RLGL, Rocha EN. Intrafamily violence and actions strategies of the Family Health team. Saúde Soc [Internet]. 2014 [acesso em 2018 Jun 05];23(3):828- 
40. Disponível em: http://www.scielo.br/pdf/sausoc/v23n3/en_0104-1290-sausoc-23-3-0828.pdf

30. Arboit J, Padoin SMM, Vieira LB, Paula CC, Costa MC, Cortes LF. Health care for women in situations of violence: discoordination of network professionals. Rev Esc Enferm USP [Internet]. 2017 [acesso em 2018 Jun 02];51:e03207. Disponível em: http://www.scielo.br/pdf/reeusp/v51/1980-220X-reeusp-51-e03207.pdf

31. Gomes NP, Erdmann AL, Bettinelli LA, Higashi GDC, Carneiro JB, Diniz NMF. The meaning of professional training for the care of women victims of domestic violence. Esc Anna Nery Rev Enferm [Internet]. 2013 [acesso em 2018 Jun 02];17(4):683-9. Disponível em: http://www.scielo.br/pdf/ean/v17n4/en_1414-8145ean-17-04-0683.pdf

32. Costa MC, Lopes MJM, Soares JSF. Violence against rural women: gender and health actions. Esc Anna Nery Rev Enferm [Internet]. 2015 [acesso em 2018 Jun 02];19(1):162-8. Disponível em: http://www.scielo.br/ pdf/ean/v19n1/en_1414-8145-ean-19-01-0162.pdf

33. Silva EB, Padoin SMM, Vianna LAC. Violence against women and care practice in the perception of the health professionals. Texto \& Contexto Enferm [Internet]. 2015 [acesso em 2018 Jun 02];24(1):229-37. Disponível em: http://www.scielo.br/pdf/tce/v24n1/0104-0707-tce-24-01-00229.pdf

34. Malta DC, Reis AAC, Jaime PC, Morais OL Neto, Silva MMA, Akerman M. O SUS e a Política Nacional de Promoção da Saúde: perspectiva resultados, avanços e desafios em tempos de crise. Ciênc Saúde Colet [Internet]. 2018 [acesso em 2019 Jun 14];23(6):1799-809. Disponível em: http://www.scielo.br/pdf/csc/ v23n6/1413-8123-csc-23-06-1799.pdf

\section{Endereço para correspondência:}

Marta Cocco da Costa

Universidade Federal de Santa Maria - UFSM

Av. Independência, 3751

Bairro: Vista Alegre

CEP: 98300-000 - Palmeira das Missões - RS

E-mail: marta.c.c@ufsm.br

Como citar: Costa MC, Silva EB, Arboit J, Honnef F, Marques KA, Barbieri J, et al. Violência doméstica contra a mulher em contexto rural: reconhecimento das estratégias de atenção. Rev Bras Promoç Saúde. 2019;32:9271. 